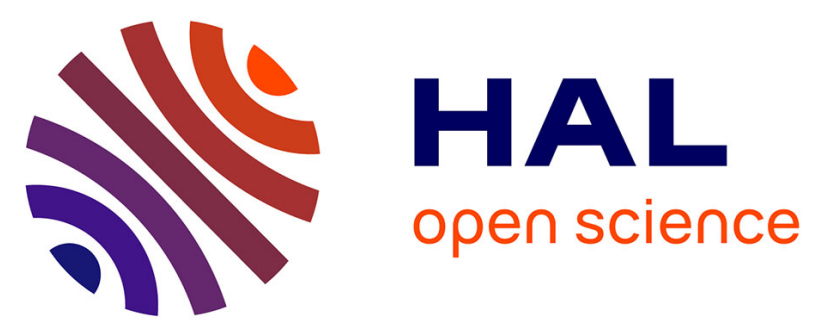

\title{
Performance of the DVB-T2 System in a Single Frequency Network: Analysis of the Distributed Alamouti Scheme
}

Nicolas Cornillet, Matthieu Crussière, Jean-François Hélard

\section{- To cite this version:}

Nicolas Cornillet, Matthieu Crussière, Jean-François Hélard. Performance of the DVB-T2 System in a Single Frequency Network: Analysis of the Distributed Alamouti Scheme. Broadband Multimedia Systems and Broadcasting (BMSB), 2011 IEEE International Symposium on, Jun 2011, Nuremberg, Germany. pp.1 - 4, 10.1109/BMSB.2011.5954961 . hal-00657495

\section{HAL Id: hal-00657495 https://hal.science/hal-00657495}

Submitted on 6 Jan 2012

HAL is a multi-disciplinary open access archive for the deposit and dissemination of scientific research documents, whether they are published or not. The documents may come from teaching and research institutions in France or abroad, or from public or private research centers.
L'archive ouverte pluridisciplinaire HAL, est destinée au dépôt et à la diffusion de documents scientifiques de niveau recherche, publiés ou non, émanant des établissements d'enseignement et de recherche français ou étrangers, des laboratoires publics ou privés. 


\title{
Performance of the DVB-T2 System in a Single Frequency Network: Analysis of the Distributed Alamouti Scheme
}

\author{
Nicolas Cornillet, Matthieu Crussière and Jean-François Hélard \\ Université Européenne de Bretagne, France \\ INSA, IETR, UMR 6164, F-35708 RENNES \\ Email: nicolas.cornillet@insa-rennes.fr
}

\begin{abstract}
The last wireless broadcast systems, like DVB-T2, have reached performances close to the Shannon limit and there remain few solutions to further increase them. One of these solutions is the use of Multiple Input Multiple Output (MIMO) techniques. In this article, we evaluate the performances of DVBT2 in a Single Frequency Network (SFN). DVB-T2 is the first broadcast standard which provides the possibility to use some Multiple Input Single Output (MISO) technique. Our study will focus on a system with two transmit antennas and one receive antenna with power imbalance between the two resulting links. We will compare the influence of this power imbalance in two configurations (MISO distributed Alamouti scheme and SISO SFN) and show the benefit brought by the use of a MISO technique in a Single Frequency Network.
\end{abstract}

\section{INTRODUCTION}

These last decades, new ways to access to multimedia contents have considerably pushed the need for high bit-rate connections. In this context, orthogonal frequency division multiplexing (OFDM) jointly used with powerful channel coding schemes like turbo-code or low density parity check codes (LDPC) is playing a central role and has already led to major performance improvements. Today, getting further in terms of transmission capacity increase essentially goes with the exploitation of the spatial component brought by multiple antenna architectures. Multiple Input Multiple Output (MIMO) techniques are indeed considered as the most promising solution to meet the needs of throughput increase and many recent telecommunication systems like IEEE 802.11 n or 3GPP-LTE already integrate such schemes.

In the domain of broadcasting, MIMO techniques are however not as well implemented as in the other telecommunication domains. The reasons for that can not only be understood by the high cost of equipping broadcasting stations with multiple antennas, but also by the fact that using MIMO schemes in a point-to-multipoint transmission scenario consisting of much contrasted reception situations is less favorable than in a point-to-point communication with link adaptation opportunities. However, many investigations on this subject are pursued and interesting proposals can be found in the recent literature [1], [2]. One first output of these research activities has in particular been proposed in the recently normalized second version of the Digital Video Broadcasting Terrestrial system (DVB-T2). Indeed, the DVB-T2 standard proposes to implement the well known Alamouti Space Time Block Code (STBC) [3] in a distributed manner across two distinct transmitters of a Single Frequency Network (SFN). In other words, the transmitter pair of the SFN amounts to a virtual single transmitter equipped with two antennas. Even if this so-called distributed Alamouti scheme is up to now only proposed as an option in the DVB-T2 standard, it can be viewed as the first incursion of multiple antenna technology in the broadcasting world.

This paper focuses on the performance of the distributed Alamouti scheme compared to a classic Single Input Single Output (SISO) transmission, in the context of a Single Frequency Network (SFN). Comparisons will be made on the basis of the DVB-T2 system specifications for various cases of spectral efficiencies. From the simulation results, we will analyze the advantages of such a scheme.

\section{SYSTEM MODEL}

\section{A. Single Frequency Network}

As explained in the introductive part, the distributed Alamouti scheme is based on an SFN topology as it can be encountered in some broadcasting networks. An SFN is an area within which a service is provided by several base stations or transmitters while exploiting the same frequency band for each station. From the receiver side, the collected signals arriving from each station can be viewed as a single signal propagated through an equivalent channel which impulse response is the sum of the impulse responses associated to each individual link. A typical example of SFN is introduced in figure 1 in the case when the SFN consists of two transmitters. In this paper, we will focus on this typical network topology.

Considering that the two transmitters are sufficiently far away from each other, it can reasonably be assumed that the signals related to each station are transmitted over two independent channels. However, since the receiver is not always located just in between the two base stations, some power imbalance factor has to be introduced between the two arriving signals. Hence, the equivalent impulse response of the SFN can be modeled as follows,

$$
h_{S F N}(\tau)=h_{1}(\tau)+\sqrt{1-\beta} \times h_{2}(\tau-\Delta)
$$



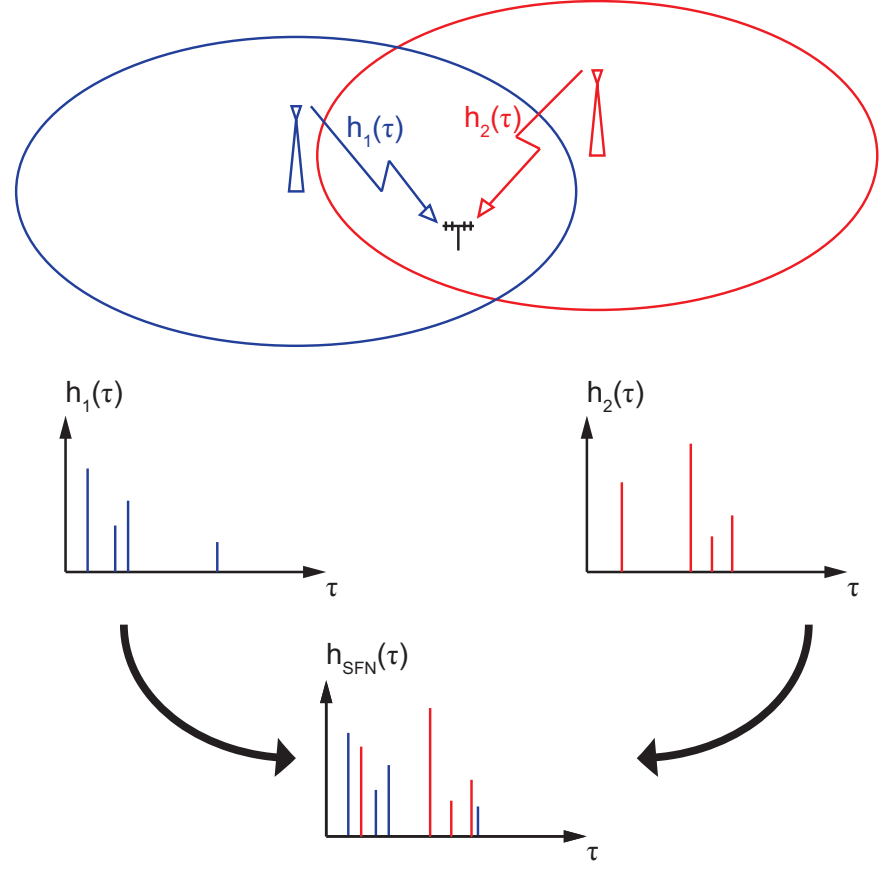

Fig. 1. Equivalent channel in a SFN with two base stations

where $h_{1}$ and $h_{2}$ are the CIR of each channel, $\Delta$ is the propagation delay to be considered between the first and second links, and $\beta$ is the attenuation factor that takes into account the link budget difference between those two links. In this paper, we will use a fixed value for $\Delta$ and focus on the influence of $\beta$. We will show that this parameter has a major influence on the performance of the system when distributed Alamouti is carried out.

\section{B. Transmission chain}

The system considered in this paper is based on the specifications of the DVB-T2 standard [4]. The implemented transmitter and receiver are depicted in figure 2 and figure 3. As evident from these figures, two transmit antennas are used to model the SFN case with two base stations, while a single antenna is exploited at the receiver side.

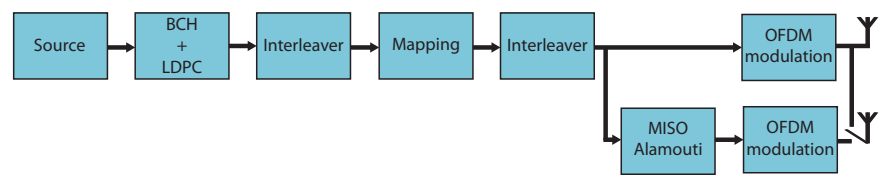

Fig. 2. Block diagram of the transmitter

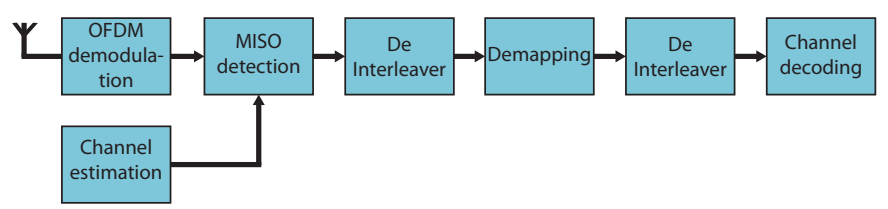

Fig. 3. Block diagram of the receiver
Considering the transmitter structure, information bits are channel encoded by a BCH encoder followed by a LDPC encoder. The encoded bits are interleaved before the mapping using rotated QAM constellations. The imaginary part of each complex symbol is then cyclically delayed before a second stage of interleaving and OFDM modulation. In the traditional SISO case, the two antennas strictly transmit the same signal. In the distributed Alamouti case however, the signal transmitted by the first antenna is the same as in the SISO case while the signal transmitted by the second antenna is encoded following the modified Alamouti scheme [3, p. 95] before the OFDM modulation.

Let $\alpha_{p, l, m}$ be the complex symbol transmitted by the $m^{\text {th }}$ antenna on the $p^{t h}$ data bearing subcarrier of the $l^{t h}$ OFDM symbol, the Alamouti scheme is defined by:

$$
\alpha_{p, l, 2}=\left\{\begin{aligned}
-\alpha_{p+1, l, 1}^{*} & \text { for } p \in\left\{0,2, \ldots, N_{D A T A}-2\right\}, \\
\alpha_{p-1, l, 1}^{*} & \text { for } p \in\left\{1,3, \ldots, N_{D A T A}-1\right\} .
\end{aligned}\right.
$$

where $N_{D A T A}$ is the number of data bearing subcarriers.

The baseband expression of the signal to be transmitted by the $m^{t h}$ antenna for the $l^{t h}$ OFDM symbol can be written as

$$
s_{l, m}(t)=\frac{5}{\sqrt{27 \times K_{T O T A L}}} \sum_{k=K_{M I N}}^{K_{M A X}} c_{k, l, m} \times \psi_{k, l}(t)
$$

where

$\psi_{k, l}(t)= \begin{cases}e^{j 2 \pi f_{k}\left(t-t_{0}(l)\right)} & \text { for } t_{0}(l)-T_{G I} \leq t \leq t_{0}(l)+T_{U}, \\ 0 & \text { otherwise, }\end{cases}$

$K_{\text {TOTAL }}$ is the number of active subcarriers, $K_{M I N}$ and $K_{M A X}$ are the indexes of the first and the last active subcarriers respectively, $f_{k}$ is the frequency of the $k^{t h}$ active subcarrier, $t_{0}(l)$ is the time of the beginning of the useful part of the $l^{\text {th }}$ OFDM symbol, $T_{U}$ is the duration of the useful part of an OFDM symbol, $T_{G I}$ is the duration of the guard interval, and $c_{k, l, m}$ is the complex modulation value for the $k^{t h}$ active subcarrier transmitted by the $m^{\text {th }}$ transmit antenna during the $l^{\text {th }}$ OFDM symbol.

At the receiver, the captured signal is first demodulated using an FFT and then applied to a MISO detector. The latter uses channel coefficients obtained from an ideal channel estimation to estimate the complex symbols that where transmitted and computes an equivalent channel coefficient for each symbol. After deinterleaving, a genie aided demapper computes log likelihood ratios (LLR) using the equivalent channel coefficients: since imaginary and real parts of each symbol are not transmitted on the same subcarrier they are not affected by the channel the same way. After a second stage of deinterleaving, the LLR are fed to the LDPC decoder and the $\mathrm{BCH}$ decoder.

\section{Simulation RESUlts}

For our simulations, we use the parameters listed in table I. The $P_{1}$ channel model used for the simulations is defined in [5] and the TU6 channel model is defined in [6]. 


\begin{tabular}{|c|c|c|}
\hline Bandwidth & \multicolumn{2}{|c|}{$8 \mathrm{MHz}$} \\
\hline FFT size & \multicolumn{2}{|c|}{$8 \mathrm{~K}$} \\
\hline Guard interval & \multicolumn{2}{|c|}{$1 / 32$} \\
\hline Pilot pattern & \multicolumn{2}{|c|}{ PP8 } \\
\hline Constellation & Rotated QPSK & Rotated 16-QAM \\
\hline Code rate & $1 / 2$ & $3 / 4$ \\
\hline Channel model & \multicolumn{2}{|c|}{$P_{1}$, TU6 } \\
\hline
\end{tabular}

Figure 4 compares the performance in SISO and in MISO on $P_{1}$ channel, with QPSK and a code rate of $1 / 2$, when the signal from the two base stations have the same power and when those signal have a $12 \mathrm{~dB}$ power imbalance. With no power imbalance, the performance of the MISO configuration is better by $0.5 \mathrm{~dB}$ compared to the SISO configuration, thanks to the diversity gain provided by the Alamouti scheme. With power imbalance, the performance of the SISO configuration remains the same. The performance of the MISO configuration is degraded by $0.9 \mathrm{~dB}$ because of noise amplification by the Alamouti decoder.

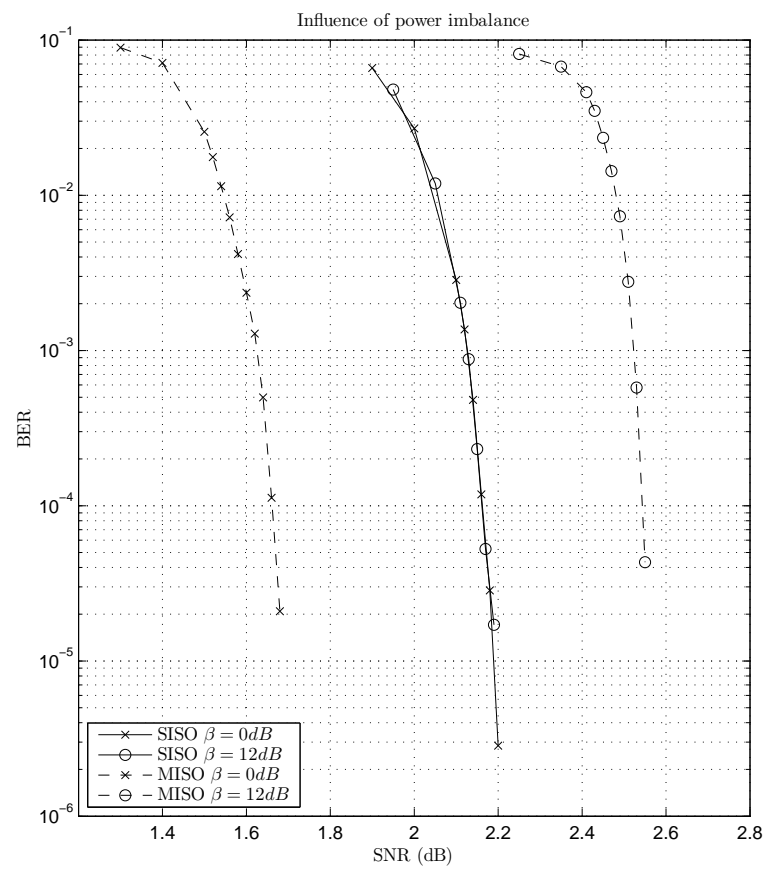

Fig. 4. Influence of power imbalance on the performance of DVB-T2 MISO and SISO transmissions with QPSK and a code rate of $1 / 2$ on $P_{1}$ channel

As shown on figure 5 results on TU6 channel are quite similar to those on $P_{1}$ channel. With no power imbalance, the performance of the MISO configuration is better by $0.4 \mathrm{~dB}$ compared to the SISO configuration. With power imbalance, the performance of the SISO configuration remains the same. The performance of the MISO configuration is degraded by $0.9 \mathrm{~dB}$.

Figure 6 shows the performance of the two configurations

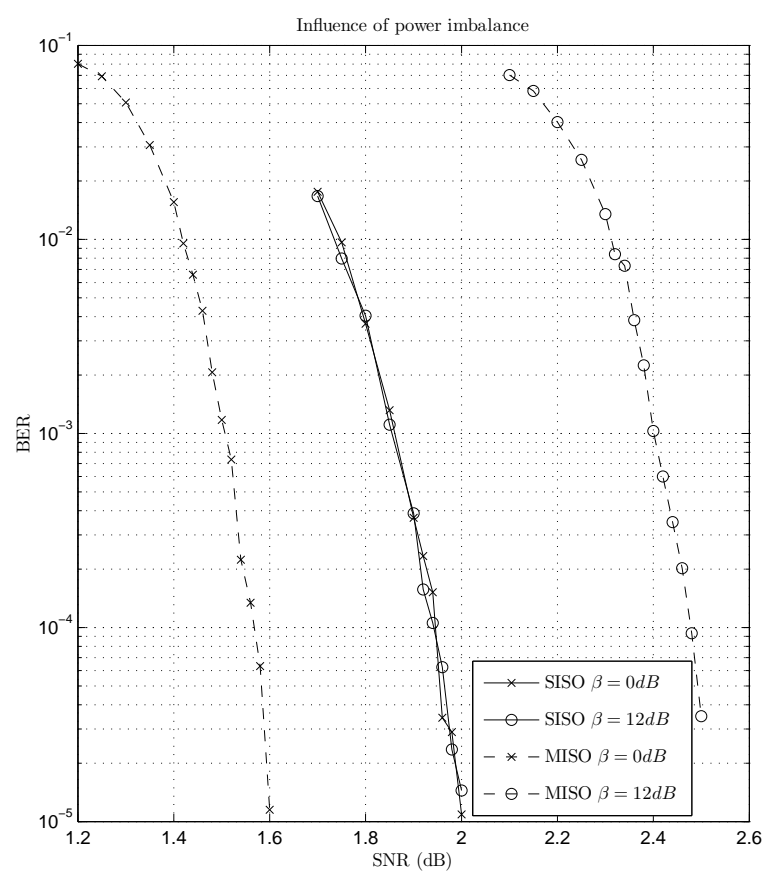

Fig. 5. Influence of power imbalance on the performance of DVB-T2 MISO and SISO transmissions with QPSK and a code rate of $1 / 2$ on TU6 channel

as a function of the power imbalance between the two base stations for a QPSK modulation and a code rate of $1 / 2$. For the P1 channel, in the SISO case, power imbalance has no influence on the system performance. A BER of $10^{-4}$ is obtained with a SNR of $2.16 \mathrm{~dB}$. In the MISO case, the required SNR varies from $1.65 \mathrm{~dB}$ to $2.54 \mathrm{~dB}$ when $\beta$ varies from $0 \mathrm{~dB}$ to $12 \mathrm{~dB}$. The MISO configuration outperforms the SISO one as far as the power imbalance stays below $6 \mathrm{~dB}$.

For the TU6 channel, the results are similar. The required SNR for a BER of $10^{-4}$ is about $1.95 \mathrm{~dB}$ in the SISO case and varies from $1.57 \mathrm{~dB}$ to $2.49 \mathrm{~dB}$ when $\beta$ varies from $0 \mathrm{~dB}$ to $12 \mathrm{~dB}$ in the MISO case. The MISO configuration outperforms the SISO one as far as the power imbalance stays below $5 \mathrm{~dB}$.

Figure 7 shows the performance with a higher spectral efficiency, i.e. 16-QAM and a code rate of 3/4 on TU6 channel. These results are quite similar to the previous ones. The required SNR for a BER of $10^{-4}$ is about $11.85 \mathrm{~dB}$ in the SISO case and varies from $11.08 \mathrm{~dB}$ to $12.37 \mathrm{~dB}$ when $\beta$ varies from $0 \mathrm{~dB}$ to $12 \mathrm{~dB}$ in the MISO case. The MISO configuration outperforms the SISO one as far as the power imbalance stays below $7 \mathrm{~dB}$.

The use of MISO for the transmission of a DVB-T2 signal in an SFN environment provides an improvement of the performance of the system. Indeed, it can be assumed that the case when the power imbalance exceeds $6 \mathrm{~dB}$ would occur in the areas where the receiver is close enough to one of the transmitter to ensure a good reception of the signal, while the case when the power imbalance is moderated corresponds to areas where the receiver is far from each station and will fully 


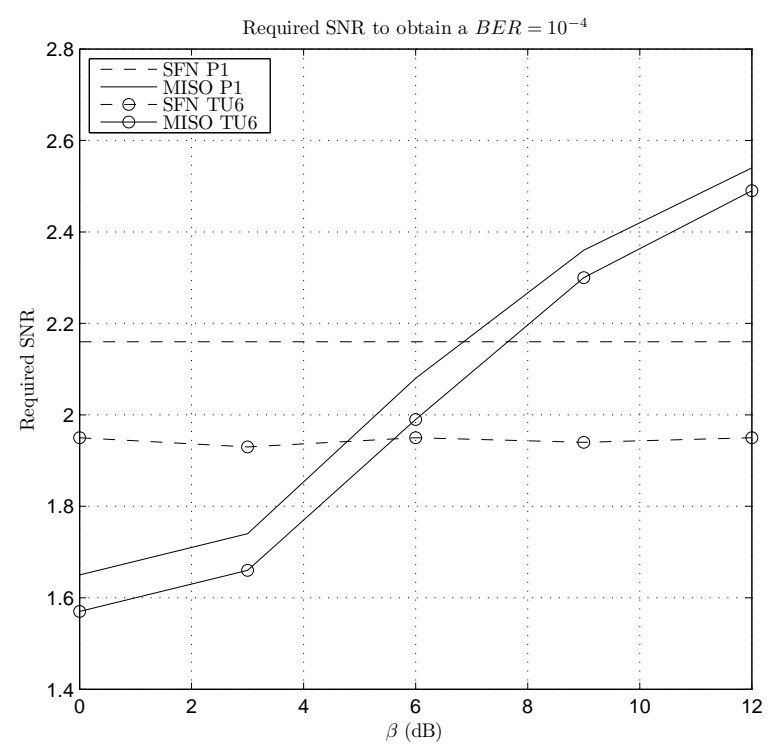

Fig. 6. Required SNR to obtain a $B E R=10^{-4}$ as a function of power imbalance for QPSK and a code rate of $1 / 2$

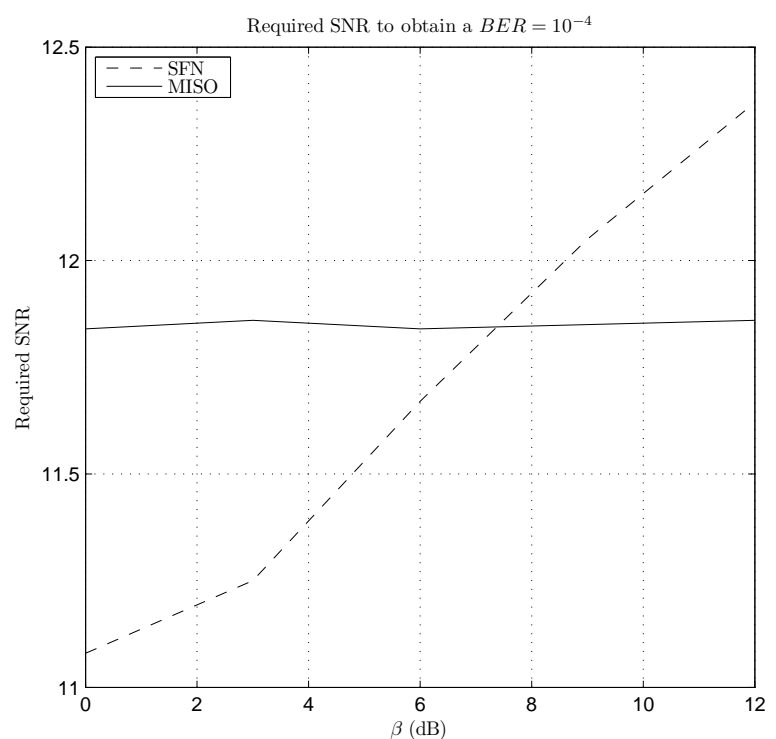

Fig. 7. Required SNR to obtain a $B E R=10^{-4}$ as a function of power imbalance for 16-QAM and a code rate of $3 / 4$ on TU6 channel

benefit from the diversity gain provided by the use of a MISO scheme.

\section{CONCLUSION}

As a first attempt to integrate multi antenna techniques in digital video broadcasting systems, the distributed Alamouti solution essentially improves the signal reception quality in SFN areas. Future work will be the study of the performance, in the same context, of other MIMO schemes such as the 3D MIMO scheme [7] which have been especially designed for the particularities of a Single Frequency Network environment.

\section{ACKNOWLEDGMENTS}

The authors would like to thank the European CELTIC project "ENGINES" for its support of this work.

\section{REFERENCES}

[1] S. Yang, L. Jianping, and C. Chaoshi, "An improved code modulation scheme with STBC for FM IBOC broadcasting," Emergency Management and Management Sciences (ICEMMS), 2010 IEEE International Conference on, pp 560-563, aug. 2010

[2] K. S. Woo, K. I. Lee, J. H. Paik, K. W. Park, W. Y. Yang, and Y. S. Cho, "A DSFBC-OFDM for a Next Generation Broadcasting System With Multiple Antennas,'IEEE transactions on broadcasting, vol. 53, no. 2,pp 539-546, jun. 2007

[3] S.M. Alamouti, "A simple transmit diversity technique for wireless communications", IEEE Journal on Selected Areas in Communications, vol. 16, no. 8, pp 1451-1458, Oct. 1998

[4] ETSI EN 302 755, "Digital Video Broadcasting (DVB); Frame structure channel coding and modulation for a second generation digital terrestrial television broadcasting system (DVB-T2)"

[5] ETSI TR 102 831, "Digital Video Broadcasting (DVB); Implementation guidelines for a second generation digital terrestrial television broadcasting system(DVB-T2)"

[6] COST 207 Report, Digital land mobile radio communications, Commission of European Communities, Directorate General, Telecommunications Information Industries and Innovation, Luxembourg, 1989.

[7] Y. Nasser, J.-F. Hélard, M. Crussière, and O. Pasquero, "Efficient 3D Space Time Space Block Code for Future Terrestrial Digital TV", Signal Processing Advances in Wireless Communications, 2008. SPAWC 2008. IEEE 9th workshop on 